\title{
On the Integrity of Data Bases and Resource Locking
}

Rudolf Bayer, Technische Universität München

\section{Abstract}

The problem of providing operational integrity of data bases as opposed to operating systems is discussed. Techniques of resource locking, mainly individual object locking and predicate locking, are surveyed, improved, and unified. An efficient on-line transitive closure algorithm for deadlock discovery is presented and analyzed. Several strategies for preventing indefinite delay of transactions are proposed. Phantoms and the need for predicate locking are surveyed and reconsidered. Several strategies for handing phantoms are proposed: one without predicate locking and two in wich predicate locking is needed for writing transactions only, and in which individual object locking sufficies for pure readers.

\section{INTRODUCTION}

Providing data base integrity means to guarantee the correctness of the data (more precisely their accuracy, consistency, and timeliness) through

1) the proper operation of the hardware,

2) the proper operation of the software, as well as

3) the proper use of the system.

This paper only covers part of the software aspect of integrity. The problem of guarding data bases against hardware failures has been covered extensively by M.V. WiIkes [WiI 72]. Proper use of the system is mainly concerned with quality control in data acquisition and with prevention of accidental or mieschievous misuse, i.e. With the security of computer systems. 
As opposed to many other computing environments, data bases give rise to especially high integrity requirements for at least the following reasons :

1) Longevity: Even rare errors will in the long run lead to a certain contamination and degradation of the quality of a data base. Completely purging erroneous data and all their consequences from a data base is ifficult.

2) Limited repeatability: Even if data or processing errors are discovered, it may be impossible or useless to rectify the situation due to time constraints, unavailability of the correct source data, unavailability of a correct system state preceding the fault.

3) The need for immediate and permanent availability: This prevents a practice often used elsewhere, namely running a program and then checking by careful inspection and analysis whether the result is or at least "looks" right, correcting and rerunning the program otherwise.

4) Multiaccess: Data bases are manipulated by many users with probably quite different quality standards. It is infeasible to completely entrust the quality control to these users and difficult to track the source and the proliteration of errors.

II. SEMANTIC AND OPERATIONAI INTEGRITY

We wish to distinguish between semantic and operational integrity of data bases:

By semantic integrity we mean the compliance of the data base contents with constraints derived from our knowledge about the meaning of the data. Semantic integrity might be enforced by allowing on certain data only a limited set of precisely specified meaningful operations, by adopting a set of programming and interaction conventions, by dynamically checking the results of updates, or by proving for each program manipulating the data base, that the semantic integrity constraints are satisfied.

Iittle is known about how to describe, to enforce, and to implement such semantic integrity constraints. Still we believe, that semantic integrity is of a much more basic nature than operational integrity, and that a better understanding of semantic integrity would greatly 
help the solution of other integrity problems as well. An approach has been described in [Bay 74] to obtain semantic integrity via the definition of "aggregates" which limit the processing of data to the use of a set of carefully designed operations directly associated with the data.

Operational Integrity: For the purpose of this discussion let a "transaction" [EGIT 74] be the unit of processing for scheduling purposes and for external data base manipulation. A transaction is a sequence of more primitive "actions". Most work to date concerned with integrity has been limited to those integrity problems arising from the activity of the operating system:

1) the effort to schedule transactions to be processed in parallel as far as possible [EGLT 74], [Eve 74], [KiC 73], [CBT 74],

2) the need to acquire resources, in particular sets of data objects or individual data objects (also called "records" in [CBT 74] and "entities" in [EGLT 74], for exclusive or shared use by a transaction and to lock those resources accordingly,

3) the induced problems of deadlock among locking transactions, of deadlock discovery, of deadlock prevention, and of preemption of tesources from transactions to resolve deadlocks.

\section{OPERATING SYSTEMS AND OPERATIONAL INTEGRITY}

As opposed to semantic integrity there is at least a brute force, straightforward solution for operational integrity, namely to avoid parallelism between transactions completely and to sequence in time the execution of transactions. This is unsatisfactory for many reasons, and better solutions have been developed for use in operating systems. We will survey these solutions briefly and indicate, why they are not satisfactory for data base applications. As usual in this field we use "process" as the analogon for "transaction". The list of techniques is adopted from G.C. Everest [Eve 74]:

Presequence Processes: Processes potentially competing for resources must be presequenced and must execute one after the other. For data base transactions it is often not known a priori, which data resources will be needed. This means that any two transactions will be potentially competing and must be sequenced. As a consequence, no parallelism is 
possible and we have the unsatisfactory brute force method mentioned before. Still presequencing transactions, e.g. through time-stamping, may be useful for other purposes, like preventing indefinite delay of transactions by introducing an aging mechanism to increase the priorities of transactions.

Preempt Processes: This technique relies on discovering deadlocks after they have occurred. It then terminates (or backs up to an earlier state) one of the processes involved in the deadlock, the resources locked by that process are freed. As we shall see, this technique plays an important role in data base locking, too, butthere its application is much more difficult due to the large number of transactions and resources involved. This makes deadock discovery and preemption quite complicated and expensive.

Preorder all System Resources: The processes are then required to claim their resources according to such a total order. It has been shown, that more general than linear orders, e.g. hierarchical orders, are sufficient to support a deadlock-free locking strategy [Ram 74]. In data bases the resources are data objects, which often do not have such an natural order. Furthermore a process might not be able to claim resources according to such an order, since his needed resources might be data dependent [EGLT 74], [CBT 74].

Preclaim needed Resources: Before starting to execute, a process has to claim all the resources it will ever need. Typically they are specified on the control cards preceding a job or job-step, and the process is not started until the operating system has granted to it all the requested resources. This is probably the most common technique for assigning non-sharable resources.

In a data base environment this technique requires considerable modifications to become feasible. Claiming resources may itself be a complicated and lengthy task requiring searching through large areas of a data base. These searches should run concurrently if possible.

Deadlock Pxevention Algorithms: They often rely on too special properties of resources - like Habermann's banker's algorithm [Hab 69] - or on too special models of computation - like Schroff's algorithm [Sch 74]to be generally applicable here. 
IV. THE CHAMBERLIN, BOYCE, TRAIGER METHOD

In [CBT 74] a technique is proposed to provide operational integrity for data bases. The technique can be considered as a modification and combination of several methods described in section III. Integrity of the data base must be guaranteed at the beginning and again at the end of a transaction, it may be - and generally must be - violated by the single actions. Due to the potential interference of two or more transactions executing in parallel, transactions must lock certain parts of the data base for exclusive or shared use. The scheme proposed in [CBT 74 ] therefore requires each transaction to lock all its resources (parts of a data base, e.g. individual records or fields of records) during a so-called "seize phase" before starting the "execution phase". During the seize phase the data base must not be modified by the seizing transaction and therefore

1) preemption of locked resources from a transaction still in its seize phase is feasible, and

2) backing a transaction in its seize phase up to wait for the preempted resource is rather easy.

Once a transaction has started its execution phase, it is not allowed to claim more resources, thus no backup will be necessary. At the end of an execution phase a transaction must free all its resources before starting a new seize phase.

The seize phase may be a rather complicated task, thus seize phases of transactions should be run in parallel. This raises the deadock problem again as usual: Let $t_{1}, t_{2}$ be two transactions. $t_{2}$ trying to seize resource $r_{1}$ already locked by $t_{1}$ must wait until $r_{1}$ is freed by $t_{1}$. But since resources are not locked in any particular order, $t_{1}$ may wish to lock first $r_{1}$, then $r_{2}$. If $t_{1}$ successfully seizes $r_{1}$ and $t_{2}$ successfully seizes $r_{2}$, then a deadlock has occurred. Such deadlocks must be discovered and a resource must be preempted from a transaction involved in the deadock, say $r_{2}$ from $t_{2}$, causing $t_{2}$ to wait for $t_{1}$ on $r_{2}$.

In [CBT 74] an aging mechanism is attached to transactions to avoid deadlock due to indefinite delay of transactions. It is then shown in [CBT 74 ] that the scheme described is deadlock-free in the sense, that each transaction will eventually be processed. This requires, of course, the proper algonithms for discovery of deadlocks between transactions in their.. seize phases, for preemption or resources, and for backing up trans- 
actions to certain points within their seize phases.

It is now clear, that the scheme proposed in [CBT 74] is a shrewd modification and combination of the following:

1) Try to preclaim needed resources.

2) If 1) would lead to deadlock, preempt resources.

3) Superimpose a presequencing scheme for transactions - e.g. through timestamping - to enforce an aging mechanism and to avoid deadlock due to indefinite delay of transactions.

V. SOME MODIFICATIONS AND AN ON-LINE TRANSITIVE CLOSURE ALGORITHM

The deadlock discovery algorithm mentioned as useful in [CBT 74] is not really applicable, since it requires that a transaction $t_{1}$ may wait for at most one other transaction to release resources. In the CBT-scheme, however, $t_{1}$ may be waiting for resources to be released by arbitrarily many transactions $t_{w_{1}}, t_{w_{2}}, \ldots t_{w_{k}}$ as the result of arbitrarily many preemptions of resources from $t_{1}$ :

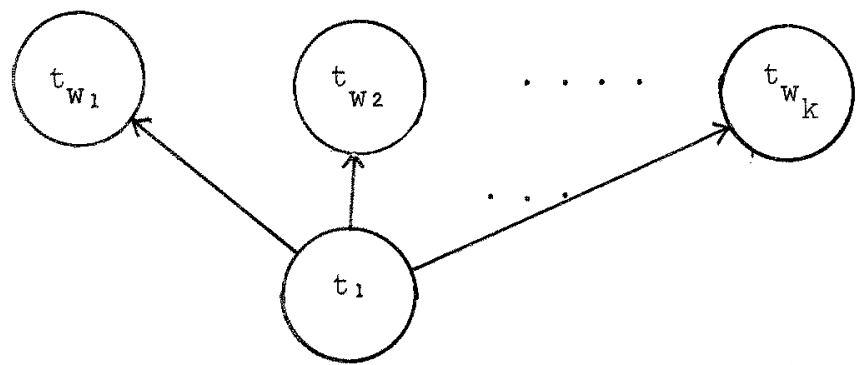

Fig. 1: Transaction $t_{1}$ waiting for other transactions.

The resource state of a transaction $t_{i}$ is determined by the set

$$
\left.A_{i}=r_{i 1}, \ldots, r_{q_{i}}\right\}
$$

of resources which it has so far acquired, and the set of request pairs

$$
B_{i}=\left\{\left(r_{i}^{2}, t_{i}\right), \ldots,\left(r_{i}^{\prime}, t_{p_{i}}, t_{p_{i}}\right)\right\}
$$

where $\left(r_{i}^{\prime}, t_{i}\right)$ indicates that resource $r_{i} j_{j}$ is desired from transaction $t_{i_{j}}$. Any transaction $t_{i}$ for which $B_{i}$ is non-empty is in a wait state. 
We may then define the wait relation wT $\times T$ where $T$ is the set of transactions, such that

$$
\left(t_{i}, t_{j}\right) \in W \text { iff } \exists r:\left(r, t_{j}\right) \in B_{i} \text {. }
$$

We say that $t_{i}$ is waiting for $t_{j}$ (to release $r$ ). $t_{i}$ may be waiting for several transactions as noted above, and for several resources from the same transaction.

The wait graph ${ }_{w}$ is the directed graph

$$
Q_{W}=(T, w)
$$

Deadlock discovery amounts to finding cycles in $G_{w}$ or, equivalently, to finding pairs ( $t, t$ ) in the transitive (but not reflexive) closure $w^{+}$ of $w$. Thus deadlock exists iff $\exists t \in T:(t, t) \in W^{+}$.

Maintaining $w$ is trivial, since something like the $B_{i}$ 's will have to be maintained in any case. Calculating $w^{+}$from $w$ is, on the other hand, quite expensive, the best known algorithms requiring $O\left(n^{3}\right)$ [War 62] or $O(n \cdot m)$ [Bay 74 ] steps, where $n$ is the number of nodes in $G_{w}$ and $m$ the number of arcs.

It would be sufficient, however, to have a good "on-line" transitive closure algorithm since $w^{+}$need only be partly modified as arcs are added to and deleted from $w$.

More precisely, "on-line" transitive closure algorithm means an algorithm solving the following problem:

$$
\begin{aligned}
& \text { Given } w, w^{+}, \quad \text { calculate } \\
& w^{\prime}, w^{+}, \quad \text { where } \\
& w^{\prime}=w \cup\left\{\left(t_{i}, t_{j}\right)\right\} \text { or } \\
& w^{\prime}=w\left\{\left(t_{i}, t_{j}\right)\right\} .
\end{aligned}
$$

Although it is quite simple to add an arbitrary arc and calculate ${ }^{+}{ }^{+}$ from $\mathrm{w}^{+}$, it seems in the general case notoriously difficult to delete an arbitrary are and calculate $w^{{ }^{+}}$from $w^{+}$. No better altemative seems to be known than calculating $\mathrm{w}^{{ }^{+}}$from scratch, i.e. starting with $w$ and ignoring the fact that we already have $w^{+}$. 
For our purpose, we need a highly simplified version of the on-line algorithm for the transitive closure only. By closer inspection one observes, that we need to delete sinks of $G_{W}$ and the arcs leading into sinks of $G_{W}$ only. This is the decisive property which makes the difficult general problem tractable in our special case. To get $w^{+}$from $w^{+}$ now simply amounts to deleting or zeroeing out a column from the Boolean matrix describing $w^{+}$.

We will now develop such an on-line transitive closure algorithm in more detail. We assume that transactions will wait in queue $q(r)$ for an already locked resource $r$. The first transaction on a queue has successfully locked (or seized) the resource, it may be in its seize or execution phase. All other transactions on the queue are waiting (or blocked). We indicate this as in Fig. 2 .

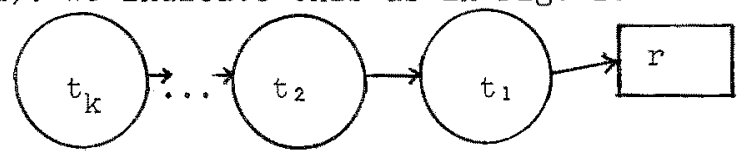

Fig. 2: Transactions waiting for resource $r$.

$t_{1}$ has locked $r$,

$t_{i+1}$ is waiting for $t_{i}$ to release (or free) $r ; i=1,2, \ldots, k-1$,

when $t_{i}$ eventually releases $r$ (and no premptions have occurred in the meantime), then $t_{i+1}$ will seize $r$.

Let us first consider the state transition diagram of a transaction (Fig. 3) and the operations relevant to that diagram, which a transaction may perform:

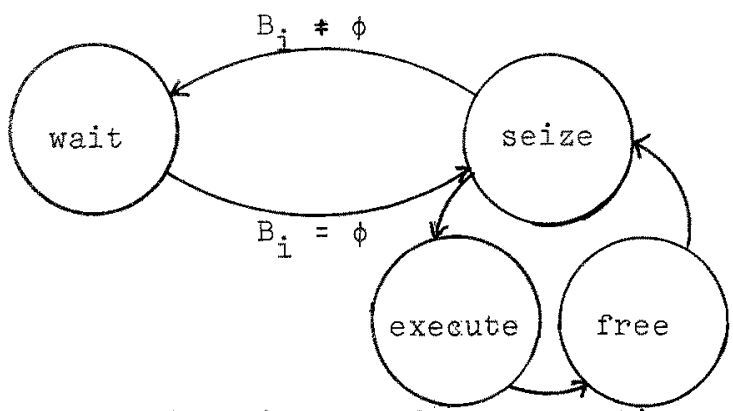

Fig. 3: The state transition diagram of a transaction.

A transaction $t_{i}$ can perform the following operations involving resource $r$ and another transaction $t_{k}$ : 
Seize r:

$A_{i}:=A_{j} \cup\{r\} ;$ update $q(r)$;

$\forall r \in A_{i}:$ Free $r: A_{i}:=\phi$;

$\forall r \in A_{i}$ do if $t_{k}$ is next in queve for $r$

then begin $\left(r, t_{i}\right)$ must be in $B_{k}$;

$B_{k}:=B_{k}-\left\{\left(r, t_{i}\right)\right\}$;

$A_{k}:=A_{k} \cup\{r\}$;

upate $q(r)$;

if $B_{k}=\phi$ then make $t_{k}$

continue to seize

end;

update $\mathrm{w}$ and $\mathrm{w}^{+}$;

Seize

unsuccessfully: $t_{i}$ is still in the seize state,

let $t_{k}$ be last in queue for $r$ :

Case 1: no deadlock arises, if $t_{i}$ is queued behind $t_{k}$ in $q(r)$ :

$B_{i}:=\left\{\left(r, t_{k}\right)\right\}$

put $t_{i}$ into wait state;

update $\mathrm{w}$ and $\mathrm{w}^{+}$;

Case 2: A deadlock would arise, if $t_{i}$ were queued as in Case 1. This deadlock is discovered by tentatively, but not definitely queueing $t_{i}$ as in case 1, updating $\mathrm{w}^{+}$and checking, whether $\mathrm{w}^{+}$contains cycles. In this case $t_{i}$ might have to preempt $r$ from $t_{k} \cdot t_{k}$ must be in wait state, since we have a cycle:

In this situation $t_{i}$ should move forward in $q(r)$ until it can be inserted and no deadlock arises; update $q(r)$ accoroingly.

Let $t_{\ell}$ be the first transaction in $q(r)$ (starting from $t_{k}$ ) such that inserting $t_{i}$ between $t_{\ell}$ and $t_{\ell-1}$ causes no deadlock, then we have Case $2 a$. If there is no such $t_{\&}$ then we have Case $2 b$.

Note: In [CBT 74] $t_{i}$ is always inserted as close to the head of the queue as possible. This strategy favors the younger transactions and must rely heavily on an aging mechanism to prevent indefinite delay. The processing costs of this aging mechanism are not analyzed. 


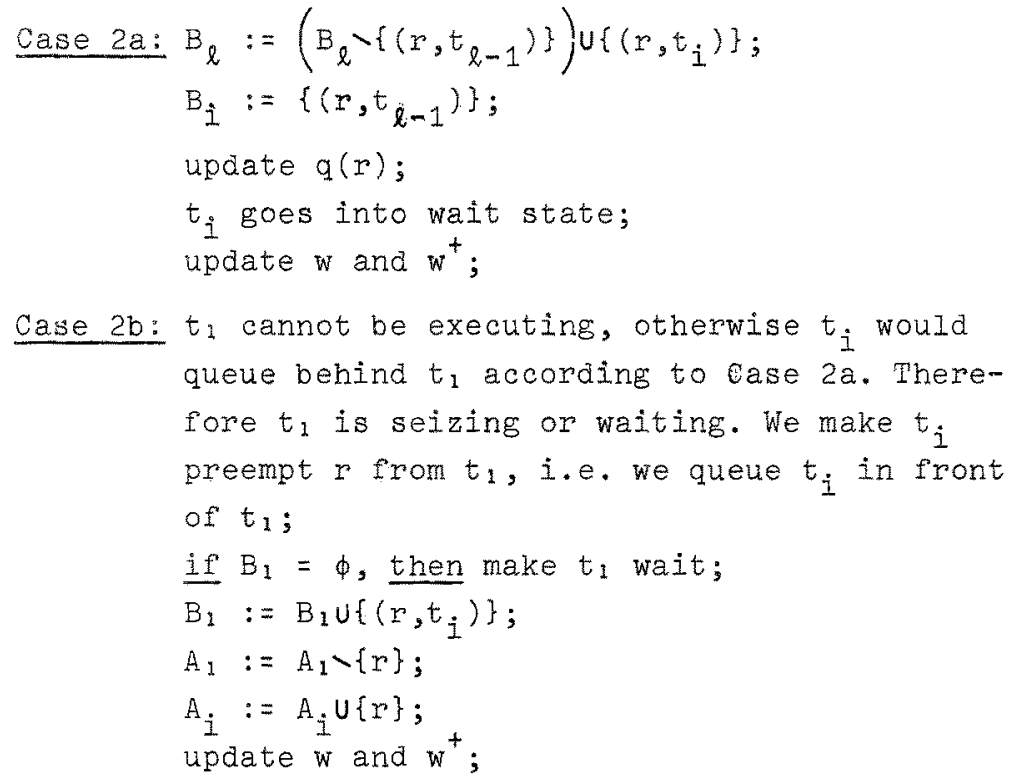

Necessary Changes to $w$ and $\mathrm{w}^{+}$and Analysis of their Complexity

For the following analysis we assume that $w^{+}$is represented in an $n \times n$ Boolean matrix $K$ with the meaning

$$
K[i, j] \equiv\left(t_{i}, t_{j}\right) \in W^{+}
$$

Operation

Description of Operation

No change to $\mathrm{w}$ or $\mathrm{w}^{+}$

Seize $r:$

$\forall r \in A_{i}:$ Free $r:$
Since $t_{i}$ frees all its resources

at the end of its execution phase,

we can remove all arcs $\left(t_{k}, t_{i}\right)$

from $w$, and delete or zero out

column $i$ of $K$.

For the analysis of the following

operations we need two auxiliary

procedures first. Let $t_{i}$ be in its

seize state. To insert an arc

$\left(t_{i}, t_{k}\right)$ into $w$ and to update $\mathrm{w}^{+}$

accordingly we need the procedure

INSERT1.
Complexity of the Change to $\mathrm{w}^{+}$

0

$o(n)$ 
To insert $\left(t_{i}, t_{i}\right)$ we need the procedure INSERT2.

INSERT1 $\left(t_{i}, t_{k}\right)$ : Comment $t_{i}$ is in seize state;

$$
\begin{aligned}
& w:=w \cup\left\{\left(t_{i}, t_{k}\right)\right\} \text {; } \\
& \text { constant } \\
& \begin{aligned}
\forall t_{j}: \quad \forall t_{\ell}: w^{+}:=w^{+} u\left\{\left(t_{j}, t_{l}\right)\right\} ; & o\left(n^{2}\right) \text { at worst, } \\
\left(t_{j}, t_{i}\right) \in W^{+}\left(t_{k}, t_{l}\right) \in W^{+} & o(m) \text { average, } \\
& \text { see lager ana- }
\end{aligned} \\
& \forall t_{\ell}: w^{+}:=w^{+} \cup\left\{\left(t_{i}, t_{\ell}\right)\right\} ; \quad \text { lysi } \\
& \left(t_{k}, t_{\ell}\right) \in W^{+} \\
& \left(t_{j}, t_{i}\right) \in w^{+}: w^{\hat{*}}:=w^{+} U\left\{\left(t_{j}, t_{k}\right)\right\} ; \quad O(n) \\
& w^{+}:=w^{+} u\left\{\left(t_{i}, t_{k}\right)\right\}
\end{aligned}
$$

INSERT2 $\left(t_{\ell}, t_{i}\right)$ : Comment $t_{i}$ is in seize state;

$$
\begin{array}{ll}
w:=w u\left\{\left(t_{\ell}, t_{i}\right)\right\} ; & \text { constant } \\
\forall t_{j}: w^{+}:=w^{+} U\left\{\left(t_{j}, t_{i}\right)\right\} ; & O(n) \\
\left(t_{j}, t_{\ell}\right) \in w^{+} & \text {constant } \\
w^{+}:=w^{+} U\left\{\left(t_{\ell}, t_{i}\right)\right\} ; & \\
\text { Note: Since } t_{i} \text { is in the seize state, } & \\
\text { there is no } t \text { such that }\left(t_{i}, t\right) \epsilon^{+} . & \\
\text {ConsequentIy no cycle in } w^{+}, \text {and } & \\
\text { therefore no deadlock can arise due } & \\
\text { to the operation INSERT2 }\left(t_{\ell}, t_{i}\right) .
\end{array}
$$

Seize $r$

unsuccessfuliy: As before, let $t_{k}$ be last in queue for $r$ :

for $j:=k$ step -1 until 1 do

begin tentatively INSERT1 $\left(t_{i}, t_{j}\right)$; if no deadlock then begin \& : $=j+1$; 
Case 2a: make last INSERT1

operation definite;

at worst

$O\left(n^{2}\right)$ or

$O(n+m)$

if $\ell \neq k+1$ then begin INSERT2 $\left(t_{\ell}, t_{i}\right) ; \quad O(n)$

$$
\begin{aligned}
& \text { if } \exists r^{\prime} \neq r^{\prime}:\left(r^{\prime}, t_{\ell-1}\right) \in B_{\ell} \text { then } \\
& \text { else } w:=w\left\{\left(t_{\ell}, t_{\ell-1}\right)\right\} \text { end; }
\end{aligned}
$$

Case 2b: INSERT2 $\left(t_{1}, t_{i}\right)$;

$O(n)$

\section{Analysis of INSERT1:}

Adding a single are to $w$, according to INSERT1, say $\left(t_{i}, t_{k}\right)$, requires oring row $k$ of the Boolean matrix $K$ to all rows $j$ with $\left(t_{j}, t_{i}\right) \in w^{+}$. At worst this part of INSERT1 requires $O\left(n^{2}\right)$ operations. If, however, there are $m$ arcs in $w^{+}$, then each node on the average will have $\mathrm{m} / \mathrm{n}$ arcs into it and $\mathrm{m} / \mathrm{n}$ arcs out of $i t$. Accordingly the average number of operations will be

$$
o(n \cdot(m / n))=o(m)
$$

VI. FOUR STRATEGIES FOR PREVENTING INDEFINITE DELAY

With the locking and preemption schemes proposed it is still conceivable, that a transaction is delayed indefinitely from its execuiton phase. To deal with this problem, we propose four increasingly effective, but also increasingly costly strategies. It seems quite reasonable to employ several strategies within one system successively in order to force transactions which have passed a certain age threshold into their execution phase and out of the system.

Strategy 1: Let $t e$ be the eldest transaction. Schedule all transactions $t$, such that $t_{e} w^{\frac{1}{t}} t$, with highest priority. This clearly has a tendency to speed up the processing of $t_{e}$. It is easy to find those $t$ from the $t_{e}$-row of the Boolean matrix describing $w^{+}$.

Strategy 2: Stop all transactions in seize phases from further seizing except those $t$ for which $t_{e}{ }^{t} t$.

Strategy 3: For all $r$ such that $t_{e}$ is waiting in $q(r)$ let $t_{x}$ be the transaction that has locked $r$. If $t_{r}$ is seizing or waiting, preempt $r$ from $t_{r}$ and give $r$ to $t_{e}$. If $t_{r}$ is executing, insert $t_{e}$ in $q(r)$ directly 
behind $t_{r}$. No new deadlocks can arise if we assume that all these preemptions are performed together in one step. Then recalculate the new $w^{+}$.

Strategy 4: Stop all transactions, which are not executing from seizing further. Then apply strategy 3 for $t_{e}$ until $t_{e}$ has reached its execution phase. Then let the other transactions proceed.

Some Oberservations on Strategies 1,2, 3, 4: It is clear that all strategies will tend to bring te closer to its execution phase.

Strategy 1 can be generalized to establish a partition of the transactions into a linearly ordered set of priority classes, which can serve as the basis for a general scheduling strategy. Strategies 1 and 3 might still allow indefinite delay. It is easy to construct a plausibility argument, that strategies 2 and 4 will prevent indefinite delay of transactions.

VII. AN ALTERNATIVE APPROACH: PREEMPTION AND PARTIAL BACKUP

Although it seems feasible to maintain the basic locking and preempting: mechanism proposed in [CBT 74] using the special algorithms described in the preceding sections, there is another argument supporting a more radical preemption than that proposed in the CBT-schene:

Let us assume that $r_{1}$ is preempted from $t_{1}$ by $t_{2}$, which probably updates $r_{1}$. Depending on the value of $r_{1}, t_{1}$ might have locked other resources $r_{1}^{\prime}, r_{1}^{\prime \prime}, \ldots$ already. But since the value of $r_{1}$ changes, the decision of $t_{1}$ to lock $r_{1}^{\prime}, r_{1}^{\prime}, \ldots$ should be reconsidered. In other words, $t_{1}$ should be backed up within its seize phase to precisely the state it was in just before seizing $r_{1}$, it should then be waiting for $t_{2}$ on $r_{1}$, and the resources $r_{1}^{\prime}, r_{1}^{\prime \prime}, \ldots$ locked by $t_{1}$ should be freed again.

In such a preemption scheme a transaction $t_{1}$ will generally be waiting for at most one other transaction $t_{2}$ on precisely one resource $r_{1}$. The wait relation $t_{1} w_{2}$ shall now mean that $t_{1}$ waits for the holder $t_{2}$ of $r_{1}$ and not for the predecessor in $q\left(r_{1}\right)$, since we do not need to maintain such queues. The resulting $G_{w}$ is obviously a forest of oriented trees, the arcs pointing towards the roots. Only roots are processing in the execution or seize phases. All other transactions are waiting. 
Since a transaction $t_{1}$ is wating for $t_{2}$ on precisely one resource $r_{1}$, we may label that arc with $r_{1}$.

The following simple algorithms then describe the necessary operations.

Seize unsuccessfully:

Case 1, no deadlock arises: $t_{1}$ trying to lock $r$ already locked by $t_{2}$ means that the tree with root $t_{1}, i . e . T\left(t_{1}\right)$, is appended as a subtree to $t_{2}$, the new arc being labelled with $r$.

Case 2, deadlock arises: Deadlock discovery is quite simple: Each seizing or executing transaction is the root node of one tree. Deadlock arises precisely when $t_{1}$ is also the root node of the tree in which $t_{2}$ is. To find this out, just follow the ares from $t_{2}$ to the root. In this case a cycle would be generated by inserting an arc $\left(t_{1}, t_{2}\right)$. The deadlock is resolved by preempting the resource $r$ from $t_{z}$.

Preemption works as follows: $t_{2}$ must free $r$ and all resources it locked after $r$. In the process - see the Free operation - corresponding subtrees of $t_{2}$ will be detached - allowing their roots to continue seizing and the arc $\left(t_{2}, t_{3}\right)$ from $t_{2}$ to its father $t_{3}$ in $T\left(t_{1}\right)$ will be deleted. The tree $T^{\prime}\left(t_{2}\right)$ remaining after pruning $T\left(t_{2}\right)$ will be attached as a subtree of $t_{1}$ by introducing the new are $\left(t_{2}, t_{1}\right)$ with label $r$.

Free $r^{\prime}:$ If $t_{2}$ frees a resource $r^{\prime}$ either due to being backed up in its seize phase or due to finishing an execution phase and there is an arc $\left(t_{4}, t_{2}\right)$ labelled $r^{\prime}$, then this arc can be deleted, thereby $t_{4}$ becomes a root and can proceed in its seize phase. To free such aros one must represent these trees by data structures in which it is possibie to follow ares in both directions.

\section{PREVENTING INDEFINITE DELAY}

It is possible that for individual transactions $t$ a situation similar to a deadlock might again arise due to t being preempted and backed up in its seize phase again and again. Strategies 1 and 2 of section VI are easily adapted to work for the preemption and backup technique of section VII. 
The analogon to strategy 3 of section VI is much easier to implement now: Let $t_{e}$ be the eldest transaction in the system again. Assume that $t_{e}$ is waiting for $t$ on $r$ and $t$ is not executing. (If $t$ is executing, nothing can be done except scheduling $t$ with highest priority until $t$ has finished executing.) Then $t_{e}$ will preempt $r$ from $t$ and $t$ will be backed up in its seize phase to a state just before seizing $r$. te becomes a root and continues seizing. A new are $\left(t, t_{e}\right)$ labelled $r$ is introduced. The preemption process works precisely as described in section VII. The main difficulty of strategy 3 of section VI has disappeared, since we do not explicitly store $w^{*}$. Instead, cycles are discovered by just following the path from an arbitrary node of a tree to its root, a simple and fast operation. To prevent pathological cases of data bases changing faster than $t$ being able to catch up in its own seize phase, we can apply an analogon to strategy 4 of section VI again. Instead, however, it suffices to prevent that transactions will enter from their seize phases into their execution phases. Let this be strategy 5. Since only finitely many transactions are in the system at any one time, and since each executing transaction will run only a finite time, $t_{e}$ will eventually be able to finish both its seize and execution phase, and indefinite delay of $t_{e}$ cannot occur.

\section{PHANTOMS AND PREDICATE LOCKS}

In [EGIT 74] a technique is described to use so called predicate locks ("predicate locking") for locking logical, i.e. existing as well as potential subsets of a data base instead of locking individual data objects ("individual object locking"). This techrique also solves the "phantom problem". To explain briefly, what phantoms are, let us assume that there is a universe $\mathcal{D}$ of data objects (called "entities" in [EGLT 74] and "records" in [CBT 741) which are the potential data objects in the data base $B$. Thus $B \subseteq D$. Two transactions $t_{1}, t_{2}$ may have successfully locked all their needed resources, and they may be executing. $t_{1}$ may add a new object $r_{1} \in D$ to $B$ and $t_{2}$ may add a new object $r_{2} \in D$ to $B$, such that $t_{1}$ would have locked $r_{2}$ and $t_{2}$ would have locked $r_{1}$, if $t_{1}$ or $t_{2}$ would have seen $r_{2}$ or $r_{1}$ resp. during their seize phases.r. $r_{1}$ and $r_{2}$ are called "phantoms", since they might, but not necessarily will appear in $B$ (materialize) while $t_{1}$ or $t_{2}$ are in their execution phases.

The appearance of just a single phantom, say $r_{1}$, does not cause any difficulty, since this has the same effect as runing the transactions $t_{1}, t_{2}$ 
serially, namely in the order $t_{2}$ followed by $t_{1}$. In this case also $t_{2}$ would not see the object $r_{1}$ created by $t_{1}$ and therefore $t_{2}$ could not be able to lock $r_{1}$. It is the goal of predicate locking to schedule transactions in parallel as far as possible under the restriction, that the paraliel schedule is equivalent to - i.e. has exactly the same total effect on the data base as - a serial schedule, One also says that such a schedule is a "consistent schedule", or that each transaction sees a "consistent view" of the data base.

To enforce consistent schedules each transaction $t$ is required to lock (for read or write access) all data objects $E(t) \subseteq D$ - imrespective of whether they are in $B$ or are just phantoms - which might in any way influence or be influenced by the effect of $t$ on $B$. $E(t)$ shall be locked by specifying a predicate $P$ defined on $D$ (or on a part of. $D$, e.g. on a relation [Cod 70]) such that $E(t) \subseteq S(P)$ where $S(P)$ is the subset of elements of $D$ satisfying $P$.

Two transactions $t_{1}, t_{2}$ are then said to be in conflict, if for their predicates $P_{1}, P_{2}$ it is true that $\operatorname{\exists r} \in S\left(P_{1}\right) \cap S\left(P_{2}\right)$ and $t_{1}$ or $t_{2}$ performs a write action on $r$. Thus conflict can arise even if $r$ is a phantom. In this case $t_{1}, t_{2}$ cannot run in parallel, but must be run serially. The order in which they are run is irrelevant for consistency. This order might be important for other reasons which are not of interest here.

The main difficulties in using such a locking and scheduling method seem to be the following:

1) Find a suitable predicate $P_{t}$ for $t$. Ideally $E(t)=S\left(P_{t}\right)$ should hold, but then $P_{t}$ might be too complicated. If $P_{t}$ is chosen in a very simple way, then $S\left(P_{t}\right)$ might be intolerably large, increasing the danger of phantoms, which are really artificial phantoms.

2) The problem " $S\left(P_{1}\right)$ nS $\left(P_{2}\right) \neq \phi$ " may be very hard. In general this problem is even undecidable. Thus for practical applications and a given it is necessary to find a suitable class of locking predicates, for Which the problem "S($\left.P_{1}\right) \cap S\left(P_{2}\right) \neq \phi$ " is not only decidable, but for which a very efficient decision procedure is known. For more details and a candidate class for suitable locking predicates see [EGLT 74].

3) Phantoms might turn out to be a very serious but mostiy artificial obstacle to parallel processing in the following sense: phantoms in 
$S\left(P_{1}\right) \cap S\left(P_{2}\right)$ prohibit $t_{1}$ and $t_{2}$ from being run in parallel. But if these phantoms do not materialize, and if furthemore $S\left(P_{1}\right) \cap S\left(P_{2}\right) \cap B=\phi$, then, of course, $t_{1}$ and $t_{2}$ could have been run in parallel. How much of an artificial obstacle phantoms are to parallel processing seems to be unknown and can probably be answered only for concrete instances of data bases.

X. A UNIFICATION OF INDIVIDUAL OBJECT LOCKING AND PREDICATE LOCKING

Let us start with the crucial observation for this section:

"Transactions, which are pure readers, do not need to lock phantoms".

A transaction is a "pure reader", if it is composed of read actions only. Obviously for many data base applications the pure readers are a very important class of transactions.

To understand our observation, consider two pure readers $t_{1}, t_{2}$ first. Since there are no write actions at all, there is no possibility for phantoms to materialize, thus they need not be locked. Phantom locking is only necessary to control the interaction with a transaction, say $t_{3}$, which also performs write operations. We call $t_{3}$ a "writer". Consider the interaction between $t_{1}$ and $t_{3}$. Let us assume that there is a phantom $r \in S\left(P_{1}\right) \cap S\left(P_{3}\right)$ such that $t_{3}$ might perform a write on $r$. Then $t_{1}$ and $t_{3}$ could not run concurrently, if $t_{1}$ would use predicate locking. If however, $t_{1}$ uses individual object locking and successfully terminates its seize phase, then $t_{1}$ can run in parallel with $t_{3}$ provided that

$$
\widehat{S\left(P_{1}\right) \cap S\left(P_{3}\right)=\phi}
$$

where $\widehat{S\left(P_{1}\right)}=S\left(P_{1}\right) \cap B$, i.e. the set of real data objects (without phantoms) in $B$ which $t_{1}$ needs to lock in order to see a consistent view of $B$. But now $\widehat{S\left(P_{1}\right)}$ can be locked by $t_{1}$ using conventional "individual object locking" as e.g. described in [CBT 74] instead of predicate locking. If $t_{3}$ should materialize phantoms, then running $t_{1}$ and $t_{3}$ in parallel still is consistent and has the same effect as the serial schedule $t_{1}$ followed by $t_{3}$.

The following observation should also be clear now: To control the interaction between the writer $t_{3}$ and the pure reader $t_{1}$ if suffices, that $t_{3}$ use individual object locking according to [CBT 74 ]. $t_{3}$ need not lock its phantoms, since $t_{1}$ is not interested in phantoms anyway. We can con- 
clude that the problem of phantoms - and therefore predicate locking arises only between writers.

The preceding observations suggest several alternative approaches for handiing the phantom problem:

Strategy 1 - Serialize Writers:

Since, as we just observed, phantoms cause difficulties only between writers, the simplest solution is, not to schedule any writers to nun concurrently. Concurrency is possible between arbitrarily many pure readers and at most one writer. Consistency is guaranteed by individual object locking and by handling deadlocks and preemptions as described in the earlier part of this paper. The problem of phantoms does not arise.

As mentioned before, in many applications most transactions are pure readers. Serializing writers in those cases should notcause a significant loss of concurrency and hes the advantage that predicate locking with its associated dificulties is not needed.

\section{Strategy 2 - Predicate Locks between Writers:}

Use predicate locks as described in [EGLT 74 ] only to determine whether two writers $t_{3}, t_{4}$ can run in parallel. After a writer is allowed to proceed on account of his predicate locks, he then starts individual object locking to compete for further processing with other transactions, which are pure readers, exactly as in strategy 1. For more details on the individual object locking phase, in particular the types of locks, see strategy 3 .

Using predicate locking and individual object locking at this point allows a more general notion of conflict than that used in [EGLT 74]. Let $U_{1}$ or $R_{1}$ be the set of objects including phantoms which are updated or only read respectively by a transaction $t_{1}$. Define $U_{2}$ and $R_{2}$ for $t_{2}$ analogously. Obviously $U_{2} \cap R_{1}=\phi$ and $U_{2} \cap R_{2}=\phi$.

Then define $B_{1}:=U_{1} \cap U_{2}$

$B_{2}:=U_{1} \cap R_{2}$

$\mathrm{B}_{3}:=\mathrm{R}_{1} \cap \mathrm{U}_{2}$

$B_{4}:=R_{1} \cap R_{2}$

Diagrammatically this can be shown as in Fig. 4. 


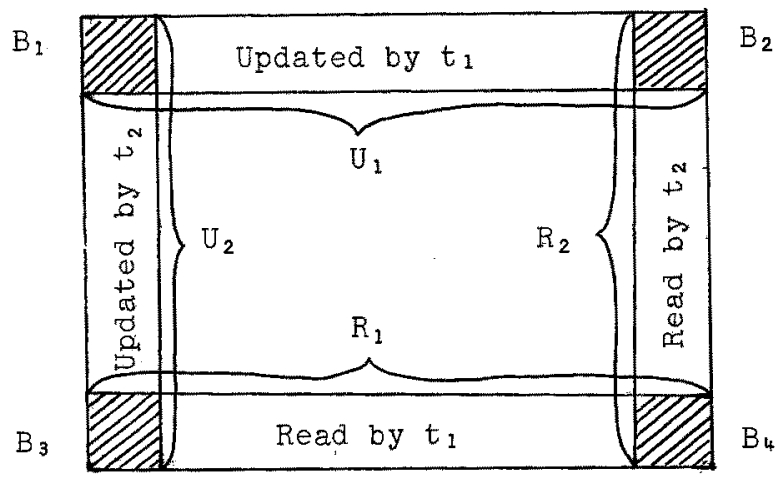

Fig. 4: Possible intersections of update and read-only sets.

For $t_{1}$ and $t_{2}$ to proceed in parallel with individual object locking the following conditions must hold:

$$
\begin{aligned}
& B_{1}=\phi \\
& B_{2}=\phi \vee B_{3}=\phi
\end{aligned}
$$

Without individual object locking the stronger condition $B_{2}=\phi \wedge B_{3}=\phi$ is required in [EGLT 74]. To see that our weakend condition suffices let us assume without loss of generality that $B_{2}=\phi$ and $B_{3} \neq \phi$.

$B_{3}$ is read only by $t_{1}$, but is updated by $t_{2}$. Also $B_{3}$ may contain phantoms which are materialized by $t_{2}$. Let us assume that both $t_{1}$ and $t_{2}$ are successful in their seize phases, i.e. while locking individual objects excluding phantoms, and then continue to run in parallel. We claim that this is equivalent to the serial schedule $t_{1}$ followed by $t_{2}$.

Since $B I$ and $B 2$ are both empty, the effect of $t 1$ on $B$ cannot in any way influence $t_{2}$, thus $t_{2}$ has the same effect on $B$ if it is run after $t_{1}$ or parallel to $t_{1}$.

$B_{3}$ is not empty, but $t_{1}$ successfully locked all the resources it needed to see a consistent view of the data base. $t_{1}$ may have missed phantoms materialized by $t_{2}$, thus the effect of $t_{1}$ will be the same as in the serial schedule $t_{1} t_{z}$. Consequently running $t_{1}$ and $t_{2}$ in parallel is equivalent to the serial schedule $t_{1} t_{2}$, and is therefore consistent.

The conditions (X.2) for $t_{1}$ and $t_{2}$ to proceed in parallel can be gener- 
alized for $t_{1}, t_{2}, \ldots, t_{n}$ to proceed concurrently. This is left to the reader.

Strategy 3: This strategy sacrifices some concurrency, but is much simpler to implement than strategy 2 . There a writer $t_{i}$ was required to perform individual object locking both in the sets $U_{i}$ and $R_{i}$. It turns out that with the conflict condition of [EGLT 74] between writers, writers need perform object locking only within $U_{i}$, but they need not set any read locks.

Assume that a writer $t_{i}$ first locks the sets $U_{i}$ and $R_{i}$ by specifying the predicates $\mathrm{P}_{U}^{i}$ and $\mathrm{P}_{\mathrm{R}}^{i}$. The condition for two writers $t_{i}$ and $t_{j}$ to run concurrently then is:

$$
\begin{aligned}
& S\left(P_{U}^{i}\right) \cap S\left(P_{U}^{j}\right)=\phi \\
& S\left(P_{U}^{i}\right) \cap S\left(P_{R}^{j}\right)=\phi \\
& S\left(P_{R}^{i}\right) \cap S\left(P_{U}^{j}\right)=\phi
\end{aligned}
$$

After successfully locking $U_{i}$ and $R_{i}$ the writer then proceeds to perform individual object locking within $U_{i}$ by setting "u-locks" for exclusive use of data objects to be updated. These u-locks are necessary for preventing pure readers from reading those objects while they are being updated. Since the sets $S\left(P_{U}^{i}\right)$ are pairwise disjoint, there is never any possibility for conflict between u-locks of different writers.

We observe that writers need not set any individual read-looks, called "r-locks", since $S\left(P_{R}^{i}\right) \cap S\left(P_{U}^{j}\right)=\phi$, and conflict of u-locks of one writer and $r$-locks of another would not be possible anyway. Furthermore, several r-locks of readers and writers on the same data object would be allowed, since data objects are shareable as long as they are only read.

The only potential conflict still remaining is between read-access of pure readers and update-access of a writer to the same data object $s$, which is not a phantom. To control this we require pure readers to set r-locks on individual data objects s to be read. This must happen during a seize phase. Several r-locks can be on $s$, but not both $r$-locks of pure readers and a u-lock of a writer. Thus if a reader (writer) sets an $r-$ lock (u-lock) first then a writer (reader) trying to set a u-lock ( $\mathrm{r}-$ lock) on the same data object must wait for the reader (writer) to release $\mathrm{s}$. This leads to the usual wait situations with the possibility for deadlock and the need for preemption and backup as described in the 
first part of this paper.

If a deadlock is discovered then a reader or a writer is backed up within its seize phase for setting r-locks or u-locks resp. as described before. For simplicity we can assume that locking with the predicates $P_{U}$ and $P_{R}$ is one indivisible operation, thus deadlock between writers is not possible during this phase of predicate locking.

To sumarize, a writer $t_{i}$ proceeds as follows:

1) Lock predicates $\mathrm{P}_{U}^{i}$ and $\mathrm{P}_{\mathrm{R}}^{i}$. If conditions (X.3) are satisfied for all other writers $t_{j}$ which have successfully locked their predicates $P_{U}^{j}$ and $P_{R}^{j}$ then proceed with step 2), otherwise wait, until $P_{U}^{i}$ and $P_{R}^{i}$ can be successfully locked, then proceed with step 2).

2) Start a seize phase setting u-locks on individual data objects to be updated within $S\left(P_{U}^{i}\right)$. In case of conflict with f-locks wait or be backed up within this seize phase.

3) A pure reader performs a seize phase setting r-locks on data objects to be read. In case of conflict with $u$-locks the reader must wait or be backed up within this seize phase.

Sumarizing the main advantages of strategy 3 we observe:

- Only writers use predicate locking to handle phantoms.

- Concurrency between writers is possible.

- Writers need an individual object locking phase for setting u-locks in their update areas only. In this phase phantoms are ignored.

- Pure readers do not use predicate locking, they set r-locks during an individual object locking phase only and ignore phantoms completely.

Note: Since predicate locking is now needed for writers only, it might be quite feasible to replace arbitrary predicates by a fixed partitioning of the data base or by a fixed family of subsets of $D$, whose interm section properties are known once and for all and recorded in a Boolean matrix (intersection between two subsets is empty or not). Instead of locking predicates the above subsets are then locked by writers.

Acknowledgement: I wish to thank Mr. John Metzgen, with whom I had many useful discussions during the writing of this paper. 


\section{Bibliography}

[Bay 74] Bayer, R., "AGGREGATES: A Software Design Method and its Application to a Family of Transitive Closure Algorithm". TUM-Math. Report No. 7432, Technische Universität München, Sept. 1974

[Bjo 73] Bjork, I.A., "Recovery Semantics for a DB/DC System". Proceedings ACM Nat'1. Conference 1973, 142-146

[CBT 74] Chamberlin, D.D., Boyce, R.F., Traiger, I.L., "A Deadlock-free Scheme for Resource Locking in a Data Base Environment". Information Processing 1974, 340-343

[Cod 70] Codd, E.F., "A Relational Model for Large Shared Data Banks". Comm. ACM 13, 6 (June 1970), 377-387

[CES 71$]$ Coffman, E.G. Jr., EIphick, M.J., Shoshani, A., "System Deadlocks". Computing Surveys 3, 2 (June 1971), 67-78

[Dav 73] Davies, C.T., "Recovery Semantics for a DB/DC System". Proceedings ACM Nat'1. Conference 1973, 136-141

[EGLT74] Eswaran, K.P., Gray, J.N., Lorie, R.A., Traiger I.L., "On the Notions of Consistency and Predicate Locks in a Data Base System". IBM Research Report RJ 1487, Dec. 30, 1974

[Eve 74] Everest, G.C., "Concurrent Update Control and Data Base Integrity". In: Data Base Management (ed. Klimbie, J.W., and Koffeman, K.L.), North Holland 1974, 241-270

[Fos 74] Fossum, B.M., "Data Base Integrity as Provided for by a Particular Data Base Management "System". In: Data Base Management (ed. Klimbie, J.W., and Koffeman, K.L.), North Holland 1974, $271-288$

[Hab 69] Habermann, A.N., "Prevention of System Deadlocks". Comm. ACM 12,7 (July 1969), 373-377, 385

[KiC 73] King, P.F., Colimeyer, A.J., "Database Sharing - an Efficient Mechanism for Supporting Concurrent Processes". AFIPS Nat'1. Comp. Conf. Proceedings 1973, 271-275 
[011 74] Olle, T.W., "Current and Future Trends in Data Base Management systems". Information Processing 1974, 998-1006

[Ram 74] Ramsperger, N., "Verringerung von Prozeßbehinderungen in Rechensystemen". Dissertation, Technische Universität München, 1974

[Sch 74] Schroff, R., "Vermeidung von totalen Verklemmungen in bewerteten Petrinetzen". Dissertation, Technische Universität München, 1974

[War 62] Warshall, S., "A Theorem on Boolean Matrices". Journal ACM 9 , 1 (January 1962), 11-12

[Wil 72] Wilkes, M.V., "On Preserving the Integrity of Data Bases". The Computer Journal, 15, 3 (August 1972), 191-194 